\title{
Wireless Sensor Network Lifetime Enhancement Using Modified Clustering and Scheduling Algorithm
}

\author{
K. Ramesh', K. Somasundaram ${ }^{2}$ \\ ${ }^{1}$ Department of IT, Nandha Engineering College, Erode, India \\ ${ }^{2}$ Department of CSE, Vel Tech High Tech Dr.Rangarajan Dr.Sakunthala Engineering College, Chennai, India \\ Email: rameshk.me@gmail.com,soms72@yahoo.com
}

Received 7 April 2016; accepted 1 May 2016; published 20 June 2016

Copyright (C) 2016 by authors and Scientific Research Publishing Inc.

This work is licensed under the Creative Commons Attribution International License (CC BY). http://creativecommons.org/licenses/by/4.0/

c) (i) Open Access

\begin{abstract}
Random distribution of sensor nodes in large scale network leads redundant nodes in the application field. Sensor nodes are with irreplaceable battery in nature, which drains the energy due to repeated collection of data and decreases network lifetime. Scheduling algorithms are the one way of addressing this issue. In proposed method, an optimized sleep scheduling used to enhance the network lifetime. While using the scheduling algorithm, the target coverage and data collection must be maintained throughout the network. In-network, aggregation method also used to remove the unwanted information in the collected data in level. Modified clustering algorithm highlights three cluster heads in each cluster which are separated by minimum distance between them. The simulation results show the $20 \%$ improvement in network lifetime, $25 \%$ improvement in throughput and $30 \%$ improvement in end to end delay.
\end{abstract}

\section{Keywords}

Clustering Algorithm, Wireless Sensor Networks, Scheduling Algorithm, Network Lifetime

\section{Introduction}

Wireless sensor network is the collection of small sensing nodes. The nodes are capable of sensing the physical parameters from application field and processing it. The processed data transferred towards base station or sink. Each node is also self-configurable with the network. In large scale network applications, the nodes are deployed randomly and also in irreplaceable conditions. So nodal energy (battery power) is to be utilized at its maximum running time. The random deployment also indicates the availability of redundant nodes in the sensing field. 
The network lifetime is the crucial factor for network design and it is directly depends on the nodes lifespan. Clustering Algorithms proposed in [1] [2] are first level for energy efficient network design. The redundant nodes may collect and transfer the repeated data and drain its energy. A scheduling algorithm is used to address this issue by considering a part of the nodes alive and others in sleep for predefined duration. Energy wastage in ideal listening of node is reduced with considerable control overhead and minimum energy loss in mode transaction (active to sleep and vice versa). A sleep scheduling algorithm without proper tuning may lead the packet loss and the network brake in the field. This degrades the network performance and also the lifetime.

In this proposal, proper tuning in scheduling algorithm is the primary goal and it considers the part of nodes as the standby nodes to provide brake free network and also to improve the packet delivery ratio. The proposed work focused on reducing energy consumption at all stages, starting from cluster formation. An optimized sleep scheduling algorithm is proposed to reduce the redundant and unwanted data transmission. Modified in-network aggregation method is used for further reduction of data transfer and its communication cast.

The rest of this paper is organized as follows. Section 2 introduces the related work for clustering, scheduling and lifetime enhancement in WSNs. Section 3 introduces the proposed system model used in this work. Section 4 is simulation results and discussion. Finally, we conclude this paper with a discussion about future research in Section 5 .

\section{Related Work}

Communication cast in direct transmission between base and nodes high and it's overcome by forming the clusters. Clusters are the group of nodes in which one of the member node is elected as leader i.e., Cluster Head (CH) [1]. CHs are elected for frequent interval in rotation manner to provide a balance network. A CH node collects the information from member nodes and sends it to sink and drain its energy quickly. So $\mathrm{CH}$ node elected for every round and it should be in rotation. Energy-efficient sensor target coverage problem was discussed in [2] for lifetime improvement.

Scheduling method is used in [3], introduces concept of sensors should power off when they are not in use for collection of information and to save energy. Energy wastages in simple ideal listening are reduced by considerable control overhead.

An approximation algorithm was developed for maximum data collection in the application field was investigated in [4]. Collected data's to reach the sink either in single hop or a multihop model based on energy level and distance between node and base. If it choose multihop to reach base, then it needs a proper routing protocol to find a path towards sink. A linear programming algorithm developed to find a shortest path to transmit data in WSNs for prolonging network lifetime in [5]. Searching tree methods used for finding the multiple path between two nodes and to select one best path to reach the base.

An energy aware organization is proposed in [6] improves the performance of the network lifetime. Maximal lifetime scheduling introduced in [7] to focus a target coverage with multiple nodes. Energy efficient targetoriented scheduling algorithm developed in [8] used for directional sensor networks and improved the performance of the network.

A comparative study of clusterhead selection algorithms in wireless sensor networks in [9] provides detailed comparison of different algorithms and its performance. Several characteristics of active/sleep models are discussed in [10] create a chain of nodes for communication hops and all sequenced nodes tend to sleep in same time to avoid the packet loss. Usually the base station is far away from the network and the nodes send the data to base using CDMA slots. Base station placement method proposed in [11] prolongs the lifetime by placing the base in Centre of the field to make a short reach to all nodes in the field.

Uneven clustering introduced in [12] improved the lifetime by avoiding overloading the nodes nearby base station. Uneven clustering concepts also prevents the hidden area called far-zone which far away from base station. Prevention of Far-zone discussed in [13] improves the network lifetime by uneven clustering and modified sleep scheduling algorithm. The proposed system optimizes the sleep scheduling and modifies the clustering algorithm to improve the network lifetime.

\section{Proposed System}

Proposed system consists of three phases: Initialization Phase, Cluster formation and $\mathrm{CH}$ selection phase and Data Relay Phase. 


\subsection{Initialization Phase}

Initialization of sensor nodes is more complex than the system design. A proper network model required the less energy consumption. Figure 1 shows the transmitter and receiver section of a node with their energy levels.

$E_{\text {elec }}$, refers to energy required to transmit or receive one bit of data. And energy required to transmit $\mathrm{n}$ bits of information for d distance with threshold $d_{0}$ is given by,

$$
\begin{gathered}
E_{T \times n}(n, d)=E_{T \times \text { Elec }}(n)+E_{T \times A m p}(n, d) \\
E_{T \times n}(n, d)=\left\{\begin{array}{l}
n E_{\text {Elec }}+n \epsilon_{f s} d^{2}, d<d_{0} \\
n E_{\text {Elec }}+n \epsilon_{m p} d^{4}, d \geq d_{0}
\end{array}\right.
\end{gathered}
$$

Free space $(f s)$ model or multipath $(m p)$ model is used based on the threshold and the distance between transmitter and receiver. To receive the above message ( $n$ bits), the receiver has to spend:

$$
E_{R \times n}(n)=n E_{\text {Elec }}
$$

Cluster head selection threshold for node $\mathrm{k}$, as follows,

$$
T(x)= \begin{cases}\frac{r E_{r e(x)}}{1-r\left(p \bmod \left(\frac{1}{r}\right)\right) E_{\max }} & n \in G \\ 0 & \text { others }\end{cases}
$$

where, $r$ is the current round, $E_{r e(x)}$ is the residual energy of the node in current round and $E_{\max }$ is the maximum energy of the a node at initial. $E_{\max }$ is common for all nodes in a homogeneous network and $\mathrm{p}$ is the probability for that node to be a cluster head.

The following pseudo-code shows initialization phase. Initially all nodes are with equal initial energy level and the threshold values. Sensor nodes are inbuilt with self-configuration and self-organizing capabilities. With this property, the nodes are initialized with the base station and update their information with neighbours. The given pseudo-code initializes all m nodes with their threshold value and finds their coordinate's values to base. A message broadcasts these information to all neighboring nodes.

\section{Pseudo-Code for Initialization Phase}
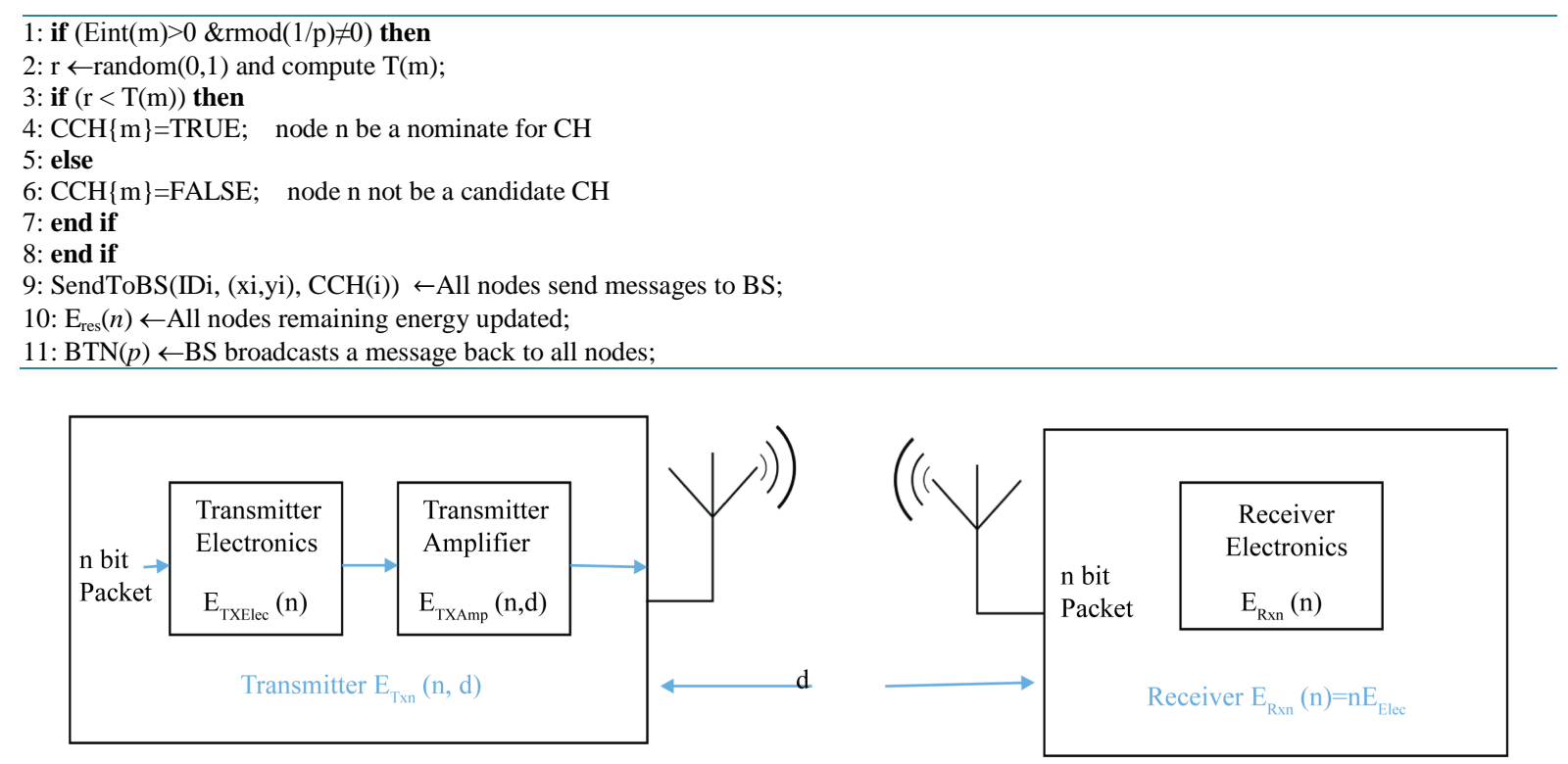

Figure 1. Radio energy model. 


\subsection{Cluster Formation Phase}

Dynamic cluster formation and Cluster Head $(\mathrm{CH})$ election is based on new threshold values $(x)$. To make a common prototype, network model follows the threshold for node $x$, as in Equation (4). If a node is eligible to act as a $\mathrm{CH}$ based on residual energy level, then its threshold value is calculated and compared with other nodes value. A node with maximum threshold value is elected as a $\mathrm{CH}$ and that node sends advertisement message to other node to join as a member of that cluster. Other nodes that receive the advertisement and are willing to join that $\mathrm{CH}$ is sent a request message to join. In this proposal three $\mathrm{CH}$ are elected within a cluster with minimum separation distance $d_{0}$. The eligible nodes before elected them self as $\mathrm{CH}$, the need to communicate to other $\mathrm{CH}$ nodes for minimum separation. If the distance is less than $d_{0}$, the system goes for an alternate, otherwise the node elected as $\mathrm{CH}$. The following pseudo-code gives the detailed $\mathrm{CH}$ election.

\section{Pseudo-Code for $\mathrm{CH}$ selection}

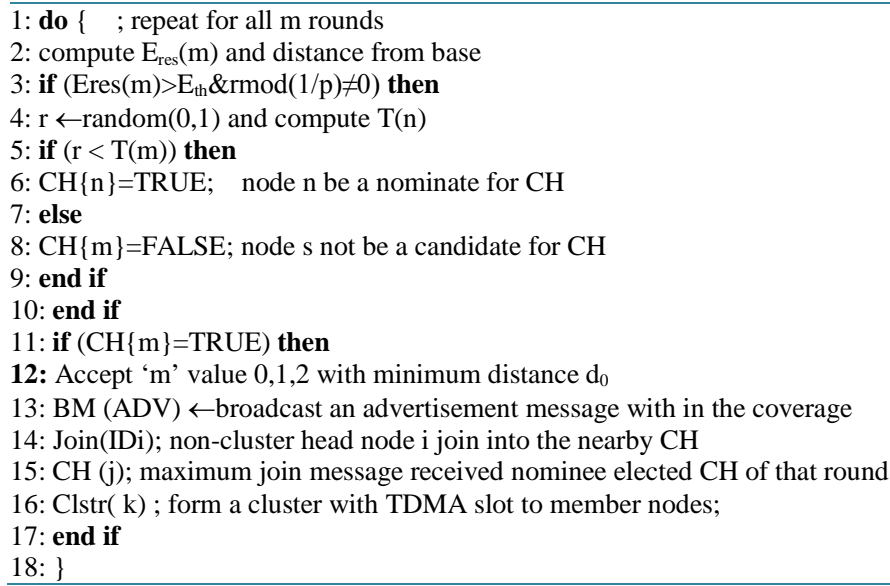

\subsection{Data Relay Phase}

In data relay phase $\mathrm{CHs}$ receive the data from members and aggregate it. Based on the distance from the base $\mathrm{CH}$ node selects the path with single hop or multiple hops to base. After selecting the path, the aggregated data with cluster head ID is transferred to next hop and maintains the hop count. If the node is a gateway node then it collects data from $\mathrm{CH}$ node and do the in-network aggregation and forward it to next hop. Member nodes are sent the sensed data in their respective time slot to base and activate sleep state.

\section{Pseudo-Code for Data Relay}

1: If $(\mathrm{CH}(\mathrm{j})=\mathrm{TRUE})$ then; repeat for all $\mathrm{CH}$ nodes

2: Receive(IDi, DataPkt) ; receive data from member nodes of that cluster $\mathrm{j}$

3: Aggregate(IDj, DataPkt)

4: Synchronize datas within Cluster's CHs.

5: TransToNh(IDj, AgDataPkt); transmit aggregated data to next hop towards base;

6: else

7: If $(\mathrm{GWN}(\mathrm{m})=\mathrm{TRUE})$ then ; gateway nodes

8: Receive(IDj, AgDataPkt)

9: InNetworkAggregation (IDj, AgDataPkt) ; In-network aggregation to remove the redundant message

10: TransToNh(IDj, AgDataPkt); transmit aggregated data to next hop towards base;

11: else

12: If (MyTimeSlot=TRUE) then; for non-CH nodes

13: TransToCH(IDi, DataPkt) ; transmit sensed data to $\mathrm{CH}$ within their time slot;

14: else

15: SleepMode(i)=TRUE ; node i at a sleep state

16: end if

17: end if

18: \} ; one round is finished

19: END 


\section{Performance Analysis}

In this section, simulation results are compared with cluster based LEACH protocol, Energy efficient targetoriented Scheduling (EETS) protocol and proposed model. The simulation parameters are 100 number of nodes with $500 \times 500 \mathrm{~m}^{2}$ area. 1 Mbps channel bandwidth, 500 bytes packet size with $1 \mathrm{~J}$ initial nodal energy are the other parameters. Performance analysis is based focused for lifetime enhancement of network with three parameters Control overhead, Packet loss, Throughput and Average Delay.

\subsection{Control Overhead}

Control overhead is the control message transaction count in specific duration. Control messages are used for having minimum bandwidth and to be reduced in overall count to avoid collision and also overall delay. Figure 2 shows the comparison of proposed protocol with other two protocols and it is considerably reduced in proposed line in number of rounds or time duration. Due to modified clustering algorithm the control overhead value reduced considerably at the later round when compared with early rounds. $20 \%$ reduction at the last round shows the improvement when compared with others.

\subsection{Packet Loss}

Packet loss and Packet delivery ratio are interrelated parameters. Packet loss is defined as the ratio between number of packets received at the end and total number of packet transmitted in specific time duration. Due to optimized sleep scheduling algorithm used in proposed method reduces the packet loss by compared with others and tends to increases the PDR value. Figure 3 shows the comparison graph and its highlights the reduced amount of packet loss in proposed method when compared with other two methods. The values are calculated in the simulation result for all the three protocols. 20\% reduction at the last round shows the improvement when compared with others.

\subsection{Throughput}

Throughput is the measure of number of packets received at receiver end in a specific time duration. Proper tuning in scheduling algorithm and reduced packet loss are helped to reach high throughput value when compared with other protocols. Comparative graph in Figure 4 shows the 25\% improvement in proposed model.

\subsection{Average End to End Delay}

The Average end to end delay is the measure of packets delayed at the receiver due to transmission in the network hop. Optimized scheduling algorithm and routing methods used in relay phase are provides reduced overall delay when compared with other protocols. Figure 5 gives comparative analysis for average delay in proposed protocol and highlights 30\% improvement in last round.

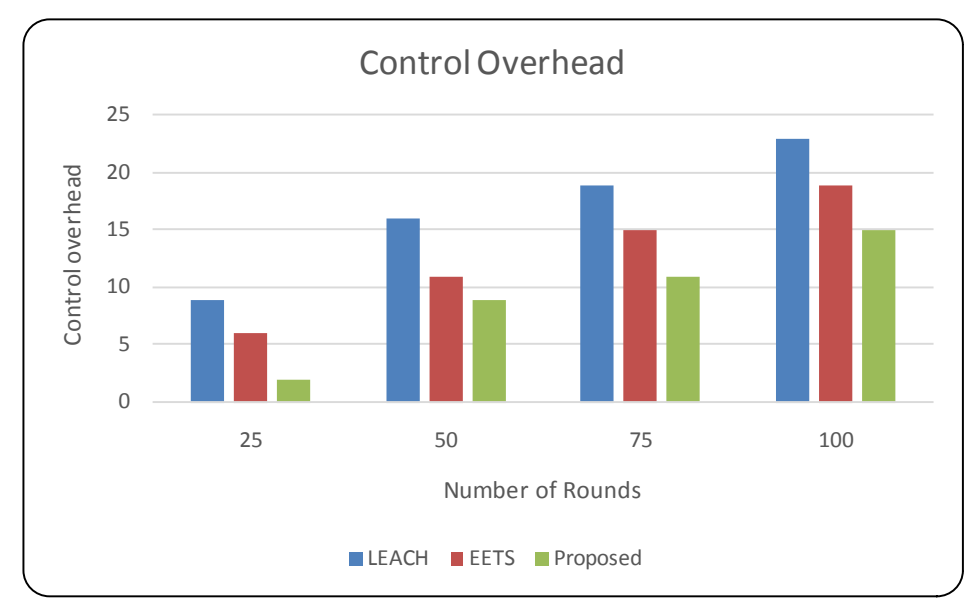

Figure 2. Control overhead comparison. 


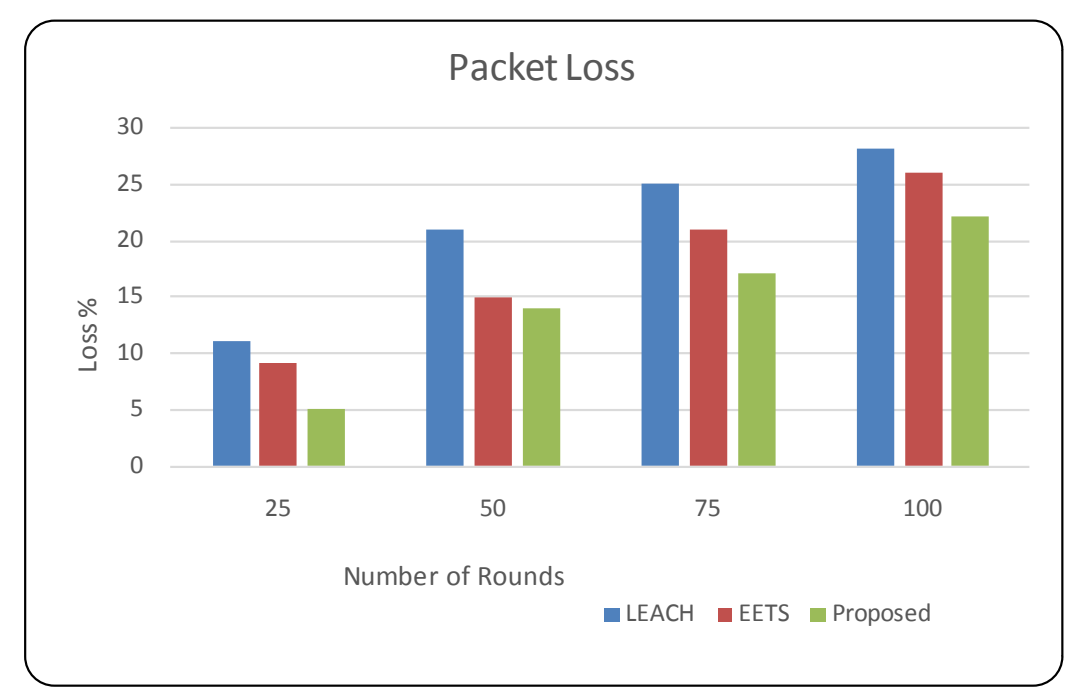

Figure 3. Packet loss comparison.

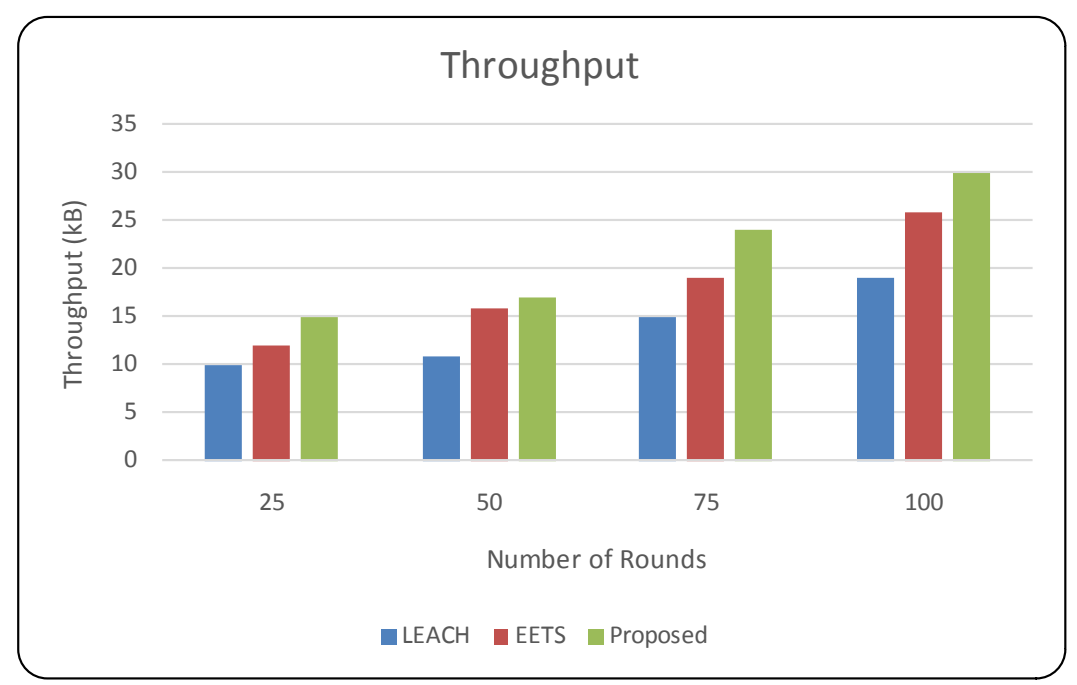

Figure 4. Throughput comparison.

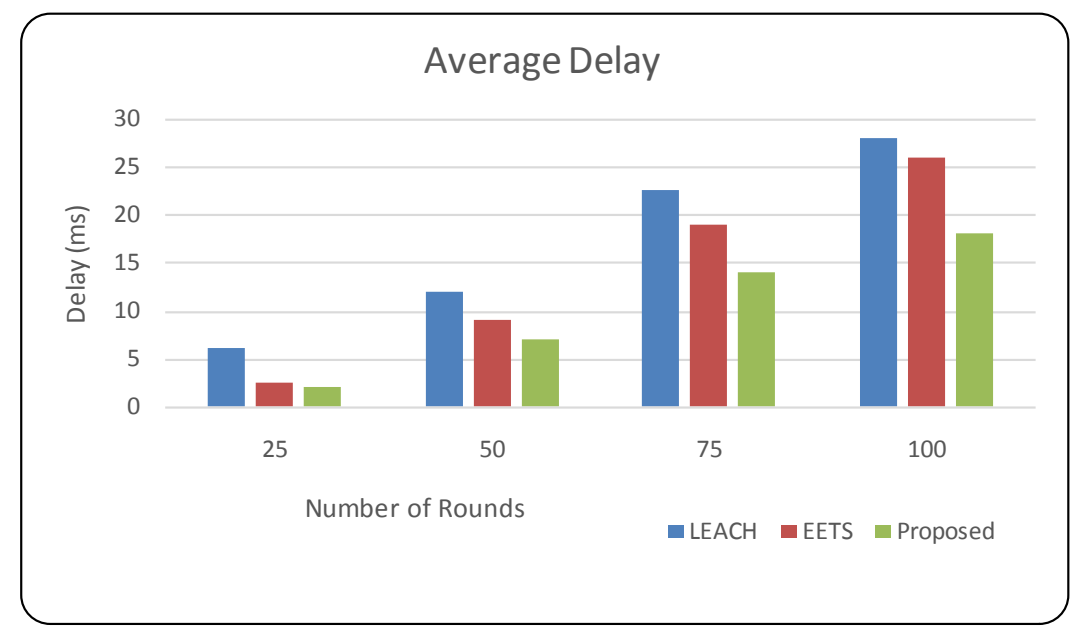

Figure 5. Average delay comparison. 


\section{Conclusion}

Prolonging wireless sensor network (WSN) lifetime is mainly by considering clustering approach and scheduling methods. The proposed protocol modifies both clustering method and sleep scheduling algorithms used in previous methods. In modified clustering, three CHs are elected within the cluster and provide load sharing between them to reduce the energy consumption for data collection and relay. In optimized Sleep scheduling model, less number of nodes in each round kept alive while others in sleep to enhance the lifetime of nodes and also the lifetime of network. 30\% improvements in overall delay and 20\% improvements in network lifetime highlight the proposed system. A modified routing protocol with minimum communication cast may enhance network lifetime as feature work.

\section{References}

[1] Heinzelman, W.R., Chandrakasan, A. and Balakrishnan, H. (2000) Energy-Efficient Communication Protocol for Wireless Sensor Networks. Proceedings of the 33rd Hawaii International Conference on System Sciences, Maui, Hawaii, 4-7 January 2000, 1-10. http://dx.doi.org/10.1109/HICSS.2000.926982

[2] Slijepcevic, S. and Potkonjak, M. (2001) Power Efficient Organization of Wireless Sensor Networks. ICC 2001 IEEE International Conference on Communication, Helsinki, 2001, 472-476. http://dx.doi.org/10.1109/icc.2001.936985

[3] Raghunathan, V., Schurgers, C., Park, S., Srivastava, M. and Shaw, B. (2002) Energy-Aware Wireless Microsensor Networks. IEEE Signal Processing Magazine, 19, 40-50. http://dx.doi.org/10.1109/79.985679

[4] Sadagopan, N. and Krishnamachari, B. (2004) Maximizing Data Extraction in Energy-Limited Sensor Networks. Twenty-Third Annual Joint Conference of the IEEE Computer and Communications Societies, Hong Kong, 7-11 March 2004, 1717-1727. http://dx.doi.org/10.1109/infcom.2004.1354583

[5] Chang, J.H. and Tassiulas, L. (2004) Maximum Lifetime Routing in Wireless Sensor Networks. IEEE/ACM Transactions on Networking, 12, 609-619. http://dx.doi.org/10.1109/TNET.2004.833122

[6] Cardei, M. and Du, D. (2005) Improving Wireless Sensor Network Lifetime through Power Aware Organization. Wireless Network, 11, 333-340. http://dx.doi.org/10.1007/s11276-005-6615-6

[7] Liu, H., Wan, P.J. and Jia, X. (2006) Maximal Lifetime Scheduling for Sensor Surveillance Systems with k Sensors to 1 Target. IEEE Transactions on Parallel and Distributed Systems, 17, 1526-1536. http://dx.doi.org/10.1109/TPDS.2006.175

[8] Cai, Y., Lou, W., Li, M. and Li, X. (2009) Energy Efficient Target-Oriented Scheduling in Directional Sensor Networks. IEEE Transactions on Computers, 58, 1259-1274. http://dx.doi.org/10.1109/TC.2009.40

[9] Ramesh, K. and Somasundaram, K. (2011) A Comparative Study of Clusterhead Selection Algorithms in Wireless Sensor Networks. International Journal of Computer Science \& Engineering Survey (IJCSES), 2, 153-164. http://dx.doi.org/10.5121/ijcses.2011.2411

[10] Li, W. (2011) Several Characteristics of Active/Sleep Model in Wireless Sensor Networks. 2011 4th IFIP International Conference on New Technologies, Mobility and Security (NTMS), Paris, 7-10 February 2011, 1-5. http://dx.doi.org/10.1109/ntms.2011.5721073

[11] Gu, Y., Pan, M. and Li, W. (2013) Prolonging the Lifetime of Large Scale Wireless Sensor Networks via Base Station Placement. 2013 IEEE 78th Vehicular Technology Conference (VTC Fall), Las Vegas, NV, 2-5 September 2013, 1-5. http://dx.doi.org/10.1109/vtcfall.2013.6692379

[12] Ramesh, K. and Somasundaram, K. (2014) Improved Energy Efficient Method for WSN to Prevent Far-Zone. Advances in Natural and Applied Sciences, 8, 1-6.

[13] Ramesh, K. and Somasundaram, K. (2014) Enhanced Energy Efficient Method for WSN to Prevent Far-Zone. International Journal on Communications Antenna and Propagation, 4, 137-142. 